\title{
The effect of implementing a new guideline and operative pro forma on the detection and management of third- and fourth-degree perineal tears
}

\author{
This article was published in the following Dove Press journal: \\ International Journal of Women's Health \\ 5 May 2016 \\ Number of times this article has been viewed
}

\author{
Kristin Cornell' \\ Alison De Souza ${ }^{2}$ \\ Mark Tacey ${ }^{3}$ \\ David M Long ${ }^{4}$ \\ Mayooran Veerasingham ${ }^{5}$ \\ 'Department of Obstetrics and \\ Gynaecology, The Northern Hospital, \\ Epping, VIC, ${ }^{2}$ Department of \\ Urogynecology, Mercy Hospital for \\ Women, Melbourne, VIC, ${ }^{3}$ Northern \\ Clinical Research Centre, The \\ Northern Hospital and Department \\ of Medicine, University of Melbourne, \\ Melbourne, VIC, ${ }^{4}$ Department of \\ Emergency Medicine, The Austin \\ Hospital, Melbourne, VIC, ${ }^{5}$ The \\ Department of Obstetrics and \\ Gynaecology, Ipswich General \\ Hospital, Ipswich, QLD, Australia
}

Background: Obstetric anal sphincter injury (OASI) is a serious complication of vaginal births, resulting in possible long-term consequences such as incontinence and pain. Adequate detection and management of these injuries is vital in minimizing the impact they have on women.

Aim: To assess the rates of detection, management, and outcomes of OASI before and after the implementation of a new clinical practice guideline and operative pro forma.

Materials and methods: A 12-month audit of the incidence, management, and outcomes of OASI was conducted in 2009. An operative pro forma and practice guideline were implemented in 2010 followed by a further audit undertaken between 2010 and 2012. Statistical analysis was performed to determine any significant change in practice.

Results: The distribution of risk factors for OASI including primiparity, birthweight, and type of vaginal delivery was similar between the two audited groups. After implementation of the pro forma, the reported incidence of OASI increased from $1.62 \%$ to $3.1 \%(P=0.004)$. Significant changes in management included an increase in the use of recommended suture material (48\% vs $80 \%, P=0.002)$, postoperative antibiotics ( $78 \%$ vs $99 \%, P=0.001)$, postoperative catheterization ( $52 \%$ vs $90 \%, P<0.001$ ), and inpatient physiotherapy consultations ( $44 \%$ vs $97 \%, P=<0.001$ ). An increase was seen in women attending their 6-week follow-up appointment (33\% vs 54\% $P=0.058)$; however, this was just below the level of statistical significance.

Conclusion: The introduction of the new pro forma and guideline resulted in an increase in the reported incidence of OASI, improved management, and follow-up of patients.

Keywords: perineal tear, obstetric anal sphincter injury (OASI), pro forma, guideline, anal incontinence, episiotomy

\section{Introduction}

Obstetric anal sphincter injury (OASI) encompasses both third- and fourth-degree perineal tears. The Australian Council on Healthcare Standards reported the rate of third-degree perineal tears was $4.7 \%$ in selected primiparous women in 2011, with fourth-degree tears occurring in less than $0.5 \%$ of these births. ${ }^{1}$ Risk factors for thirdand fourth-degree tears include nulliparity, instrumental delivery, midline episiotomy, delayed second stage of labor, and birthweight over $4 \mathrm{~kg} .^{2}$

Women with clinically evident sphincter tears are significantly more likely to report fecal incontinence, fecal urgency, and flatal incontinence compared to women without sphincter tears. ${ }^{3}$ The prevalence of anal incontinence following primary repair of OASI is poorly defined with estimates between $15 \%$ and $61 \%$. $^{4}$ Incontinence is
Correspondence: Kristin Cornell The Royal Women's Hospital, 20 Flemington Road, Parkville, Melbourne, VIC, 3052, Australia

Tel +6I 383452000

Email kristin.cornell@thewomens.org.au
International Journal of Women's Health 2016:8 131-135

(c) (1) \& () 2016 Cornell et al. This work is published and licensed by Dove Medical Press Limited. The full terms of this license are available at https://www.dovepress.com/terms.php (c) hereby accept the Terms. Non-commercial uses of the work are permitted without any further permission from Dove Medical Press Limited, provided the work is properly attributed. For permission for commercial use of this work, please see paragraphs 4.2 and 5 of our Terms (https://www.dovepress.com/terms.php.
Dovepress

http://dx.doi.org/10.2147/1]WH.SI01188

. 
more common in women with fourth-degree $(25.0 \%)$ than with third-degree tears $(11.5 \%) .^{5}$

Poor repair technique and inadequate obstetric training has been cited as major contributing factors for anal incontinence. ${ }^{6}$ Uppal et al found that $60 \%$ of obstetric residents had not had any didactic teaching on perineal repair and no formal teaching on pelvic floor anatomy. ${ }^{7}$ The level of training in recognition and management of OASI for medical and nursing staff is often limited. Misdiagnosis of tears, poor repair technique, or inadequate postoperative management may all contribute to poor outcomes.

An initial 12-month audit conducted at our hospital found significant areas for improvement in the management of OASI. We hypothesized that the implementation of an operative pro forma and clinical guideline to be completed for every third- and fourth-degree tear would improve the rate of detection and management of patients with OASI.

\section{Materials and methods}

The study was conducted at The Northern Hospital (Melbourne, Australia) between 2009 and 2012. The Northern Hospital is a level 2 metropolitan hospital with approximately 2,500 deliveries per year. A retrospective 12-month audit was conducted in 2009 examining the incidence and management of OASI. The information collected included: maternal age and parity, mode of delivery, neonatal birthweight, degree of OASI, and use of episiotomy. Data were obtained using the birthing outcome system, maternity discharge summaries, and the hospital patient record. The management of each tear was further investigated including:

- the level of experience of the surgeon present at the repair;

- the location in which the repair took place (birthing suite or the operating theater);

- the repair technique;

- the suture material used;

- mode of anesthesia;

- the use of antibiotics, catheters, aperients, and analgesia;

- subsequent follow-up.

Follow-up was assessed by analyzing physiotherapy and obstetric consultations. The retrospective audit group is referred to as group 1.

Following the analysis, deficiencies in the management of OASI were identified and an operative pro forma (Supplementary material 1) was designed along with a protocol for managing third- and fourth-degree tears (Supplementary materials 2). The operative pro forma was a "tick box" worksheet requiring adequate assessment of the tear and other management prompts.
The protocol gave specific directions regarding repair, and immediate and short-term management. The protocol and pro forma were implemented on the ward for a period of 18 months, during which time a second audit was performed from August 2010 to February 2012 (herein group 2).

Approval to conduct the analysis was granted by the Northern Health Low and Negligible Risk Ethics Committee (approval number ALR 04.2014). As the study was an audit in relation to improvements in clinical practice there was not a requirement for patient consent.

Descriptive statistics were prepared in order to compare the two groups. For continuous, normally distributed variables such as age and birthweight, a Student's $t$-test was used to test for significant differences between the two groups. The chi-squared test and Fisher's exact test were used to test for differences across groups for categorical variables. All tests were twotailed, and a $P$-value $<0.05$ was considered to indicate statistical significance. Statistical analysis was performed using Stata version 12.1 (StataCorp LP, College Station, TX, USA).

\section{Results}

In group 1, 1,668 women delivered vaginally with 27 (1.6\%) OASI reported. In group 2, the total number of vaginal deliveries was 2,634, with 82 (3.1\%) OASI reported, nearly double that of the previous audit $(P=0.004)$. There was a $68 \%$ compliance rate with the pro forma.

The average age ( \pm standard deviation) was similar across the two groups (group 1: $26.3 \pm 5.5$ vs group 2: 28.0 \pm 4.4 , $P=0.113$ ). The majority of women affected were primiparous ( $81 \%$ in group 1 and $84 \%$ in group $2, P=0.805$ ). There was no difference in the average ( \pm standard deviation) birthweight across the two groups (group 1: $3,373 \mathrm{~g} \pm 85.9 \mathrm{~g}$ vs group 2: $3,367 \mathrm{~g} \pm 56.8 \mathrm{~g}, P=0.953)$ with a majority of OASI occurring during the delivery of babies in the 3,100-3,500 g group in both audits, comprising $52 \%$ of tears in group 1, and $44 \%$ in group 2.

The proportion of $3 \mathrm{a}$ and $3 \mathrm{~b}$ degree tears in group 2 was slightly higher when compared to group $1(P=0.280)$, although this difference was not statistically significant (Table 1).

Table I Distribution of the types of perineal tear within each group; percentage (\%) and number of patients (n)

\begin{tabular}{lll}
\hline Degree of tear & $\begin{array}{l}\text { Group I } \\
(\mathbf{n = 2 7 )}\end{array}$ & $\begin{array}{l}\text { Group 2 } \\
(\mathbf{n = 8 2 )}\end{array}$ \\
\hline 3a & $29 \%(8)$ & $39 \%(3 \mathrm{I})$ \\
3b & $37 \%(10)$ & $43 \%(34)$ \\
3c & $19 \%(5)$ & $10 \%(8)$ \\
4th degree & $11 \%(3)$ & $8 \%(6)$ \\
Buttonhole tear & $4 \%(1)$ & $0 \%(0)$ \\
\hline
\end{tabular}


Fifty-three percentage of OASI occurred with normal vaginal births, with no significant difference across groups (group 1: $56 \%$ vs group 2: $52 \%, P=0.465$ ). The remaining variables listed on the pro forma were then analyzed (Table 2 ). There was no difference in OASI between groups in patients receiving an episiotomy ( $74 \%$ vs $66 \%, P=0.428$ ).

A senior clinician was present in $63 \%$ of repairs in group 1, and $75 \%$ in group 2, and this difference was not significant $(P=0.243)$. There was, however, a significant difference in the proportion of cases where a consultant was present, with $59 \%$ in group 1 and $27 \%$ in group $2(P=0.002)$. Sixty-three percentage of patients in both groups were transferred to the operating theater for repair of the OASI.

There was no significant change in the number of patients receiving regional anesthesia for the repair of their OASI ( $70 \%$ vs $73 \% P=0.759$ ). A significant improvement was seen between groups with use of polydioxanone (PDS) for the repair of the external anal sphincter ( $48 \%$ vs $80 \%, P=0.002$ ). In group $2,40 \%$ of OASI were repaired end-to-end, with $51 \%$ overlapping and $9 \%$ not stated. This information was not available for analysis in group 1.

More patients in group 2 received intravenous antibiotics postoperatively ( $99 \%$ vs $78 \%$ ) with $51 \%$ receiving them for 48 hours.

The rate of documented postoperative catheterization improved from $52 \%$ to $95 \%(P<0.001)$. All patients in group 1 received aperients and this continued following introduction of the guideline. Inpatient physiotherapy consultation increased from $44 \%$ to $97 \%(P<0.001)$ from group 1 to group 2, and although 6-week follow-up attendance improved, this was not statistically significant (33\% vs $54 \%, P=0.058)$.

Our protocol advised the use of non-constipating analgesia such as tramadol, and regular paracetamol and diclofenac. This was not audited in group 1; however in group 2 , only $18 \%$ of

Table 2 Audited variables, \% (n)

\begin{tabular}{llll}
\hline & $\begin{array}{l}\text { Group I } \\
(\mathbf{n = 2 7 )}\end{array}$ & $\begin{array}{l}\text { Group 2 } \\
(\mathbf{n}=\mathbf{7 9})\end{array}$ & P-value \\
\hline Episiotomy cut & $74(20)$ & $66(52)$ & 0.428 \\
$\begin{array}{l}\text { Senior clinician present } \\
\text { (consultant or senior registrar) }\end{array}$ & $63(17)$ & $75(59)$ & 0.243 \\
$\begin{array}{l}\text { Repair carried out in theater } \\
\text { Regional or general anesthesia }\end{array}$ & $63(17)$ & $63(50)$ & 0.976 \\
Monofilament used & $48(19)$ & $73(58)$ & 0.759 \\
Postoperative antibiotics & $78(21)$ & $80(63)$ & 0.002 \\
Postoperative catheterization & $52(14)$ & $95(78)$ & 0.001 \\
Postoperative aperients & $100(25)$ & $100(79)$ & $<0.00$ I \\
Inpatient physiotherapy & $44(12)$ & $97(77)$ & $<0.000$ \\
consult & & & \\
Six-week follow-up & $33(9)$ & $54(43)$ & 0.058 \\
\hline
\end{tabular}

women received tramadol in addition to paracetamol and diclofenac. Fifty percentage received additional oxycodone and $32 \%$ received only paracetamol and diclofenac.

Twenty-five percentage of patients in group 1 were questioned about their anal continence at follow-up and of these, all women reported full continence. All patients in group 2 who attended follow-up also reported full fecal and flatal continence. One patient with a fourth-degree tear was experiencing symptoms of fecal incontinence.

\section{Discussion}

We found the implementation of a clinical guideline describing best practice for the repair of OASI and postoperative management improved the postoperative and postnatal care of women following delivery. In addition, we observed a significant increase in the number of OASI detected and reported at our institution after the introduction of the new operative pro forma.

The rate of OASI reported at our institution increased between audited groups from $1.6 \%$ to $3.1 \%$. The figure of $3.1 \%$ in group 2 was more consistent with Australasian published data at the time of this study and also with comparable institutions. It is difficult to identify the true explanation for the differences between groups. One possibility is that the change was entirely due to chance. Another interpretation is that prior to the introduction of the pro forma, the rate of detection at this institution was relatively poor, and that improved education led to an increase in reporting and therefore an increase in the rate of detected OASI.

It has been previously shown that increased awareness and training is linked with an increase in the detection of sphincter injuries. ${ }^{8}$ There was no difference in risk factors for OASI between groups, or changes in obstetric protocols between pre- and post-pro forma implementation to account for this rise. Specifically, risk factors such as operative delivery remained the same over both time frames $(9.5 \%$ of deliveries in group 1, and $11 \%$ in group 2). A decline in the rate of unclassified tears in group 2 may have been more correctly identified as OASI. This is significant as increased vigilance improves the clinical diagnosis of OASI. ${ }^{8}$

We believe that this highlights the need for improvements in training of midwives and doctors in perineal anatomy and recognition of OASI. Improvements in diagnosis allow appropriate repair that may lead to improvements in longterm outcome.

The literature suggests that the level of clinician present at an OASI repair can affect patient outcomes. Anal symptoms secondary to OASI may be related to inexperienced clinicians assessing and repairing these tears. ${ }^{6}$ In our study, there was 
a higher rate of senior clinicians attending and supervising the OASI repair in group 2 with no repairs performed by an unsupervised junior RMO (resident medical officer).

The level of training of the registrars performing repairs in our study was often not specified. Variation in experience can be significant with some trainees having attended dedicated OASI courses compared to unaccredited trainees with minimal teaching. An audit by Andrews et al showed that only $13 \%$ of obstetric trainees were satisfied with their level of experience prior to performing their first unsupervised repair. ${ }^{9}$ Increased consistency in the quality of the repair may help to improve outcomes. This could include mandatory attendance at an OASI workshop prior to independently repairing an OASI and 6-monthly training sessions for all midwives and medical staff.

Optimal conditions for conducting a repair are in the operating theater, with an aseptic environment, adequate lighting, exposure, equipment, and regional anesthesia. Regional or general anesthesia allows relaxation of the anal sphincter, optimizing the repair. ${ }^{10}$ The location of the repair remained unchanged over both groups with $63 \%$ being repaired in the operating theater. Limitations to theater access remained a barrier to improving this figure as there was no dedicated maternity theater at this hospital. Compulsory repair of any OASI in theater is becoming standard practice at most hospitals and should be considered essential in the ideal management of these injuries.

The current literature supports either monofilament or braided suture material for repair of the endoanal sphincter. Monofilament suture (PDS) was selected as our preferred material for the guideline as it is thought to be less likely to harbor organisms, ${ }^{11}$ and therefore decrease the risk of wound infection. A significant increase in the use of PDS was noted following the introduction of our guideline.

In terms of technique, both end-to-end and overlapping methods were acceptable in the guideline, consistent with a Cochrane review in 2010, which found insufficient evidence to recommend one over the other. ${ }^{12}$ Both methods were used by our clinicians in similar numbers.

Antibiotics are generally recommended to reduce the risk of perineal wound complications such as infection, breakdown, and development of fistulae. ${ }^{13}$ Intra-operative second-generation cephalosporins have been shown to significantly reduce perineal wound infections from $24.2 \%$ to $8.2 \% .{ }^{14}$ We recommended $24-48$ hours of cefazolin and metronidazole. The rates of compliance with intra- and postoperative antibiotics were all improved after implementation of the new guideline.
Bladder sensation takes approximately 6 hours to return after regional anesthesia, and after an OASI, perineal discomfort can increase in this period to up to 12 hours, ${ }^{15}$ increasing the risk of urinary retention. The low rates of catheterization seen in group 1 improved markedly in group 2 .

Attempts to minimize the use of traditional opioids were challenging with the majority still being prescribed drugs such as oxycodone rather than potentially less constipating agents such as tramadol. It is not clear why this was the case and may reflect lack of education of junior staff regarding postoperative analgesic options. At the time of this study, the oxycodone/naloxone hydrochloride (Targin) combination was not available. With its lower constipating profile, Targin maybe a better postoperative analgesic in this patient group. Aperients were routinely used in all patients having undergone OASI repair.

Follow-up of women sustaining OASI remained poor despite the intervention; in group 1, only 33\% of women attended their 6-week checkup, with only a marginal improvement in the second group (54\%). This may have been due to a lack of understanding by patients of the importance of follow-up to discuss symptoms, be examined, and plan future deliveries. Those patients who did attend follow-up appointments were almost all asymptomatic, suggesting that patients who are well are more motivated to attend. Poor postnatal attendance can be due to a multitude of reasons including lack of transport, fatigue, an unsettled or unwell baby. We suggest that these women be followed up by phone contact from a hospital midwife and/or offered a further appointment by mail to ensure they have had adequate outcomes.

\section{Limitations}

There were several limitations to this study. There was only a $68 \%$ compliance rate with the pro forma, and while there was formal education provided to the staff initially, this was not regularly performed throughout the time of the audit. The sample group may not be representative of pregnant women elsewhere in Victoria. The Northern Hospital has a very diverse catchment of cultures, which was not controlled for. The benefit of the intervention was also difficult to interpret due to the small number of patients who attended follow-up.

\section{Conclusion}

Our study has shown improved reporting and management of OASI with the introduction of the operative pro forma and guideline. We recommend the implementation of the operative pro forma and guideline in similar institutions to 
enhance best practice in the management of OASI. Further research and follow-up of patients are needed to determine subjective and objective outcomes of patients following OASI repair with the use of the guideline.

\section{Disclosure}

The authors report no conflicts of interest in this work.

\section{References}

1. Australian Council on Healthcare Standards, Obstetrics version 7.2 ACHS Clinical Indicator User Manual 2013:9-10. Available from: http:// www.achs.org.au/media/88679/clinical_indicator_report_2006_2013. pdf. Accessed March 72016.

2. Royal College of Obstetricians and Gynaecologists Green-top guideline No 29. The management of third and fourth degree perineal tears. UK: RCOG; 2007. Available from: https://www.rcog.org.uk/globalassets/ documents/guidelines/gtg2911022011.pdf. Accessed February 21, 2016.

3. Borello-France D, Burgio KL, Richter HE, et al. Fecal and urinary incontinence in primiparous women. Obstet Gynecol. 2006;108(4): 863-872.

4. Samarasekera DN, Bekhit M, Wright Y, et al. Long-term anal continence and quality of life following postpartum anal sphincter injury. Colorectal Dis. 2008;10(8):793-799.

5. Sangalli MR, Floris L, Faltin D, Weil A. Anal incontinence in women with third or fourth degree perineal tears and subsequent vaginal deliveries. Aust N Z J Obstet Gynaecol. 2000;40(3):244-248.
6. Thakar R, Sultan AH, Fernando R, Monga A, Stanton S. Can workshops on obstetric anal sphincter rupture change practice? Int Urogynecol J. 2001;12(3):S5.

7. Uppal S, Harmanli O, Rowland J, Hernandez E, Dandolu V. Resident competency in obstetric anal sphincter laceration repair. Obstet Gynaecol. 2010;115(2 Pt 1):305-309.

8. Groom KM, Patterson-Brown S. Can we improve on the diagnosis of third degree tears? Eur J Obstet Gynecol Reprod Biol. 2002;101(1): 19-21.

9. Andrews V, Thakar R, Sultan A. Structured hands-on training in repair of obstetric anal sphincter injuries (OASIS): an audit of clinical practice. Int Urogynecol J Pelvic Floor Dysfunct. 2009;20(2):193-199.

10. Walsh CJ, Mooney EF, Upton GJ, Motson RW. Incidence of third-degree perineal tears in labour and outcome after primary repair. Br J Surg. 1996;83(2):218-221.

11. Katz S, Izhar M, Mirelman D. Bacterial adherence to surgical sutures. A possible factor in suture induced infection. Ann Surg. 1981;194(1): 35-41.

12. Fernando FJ, Sultan A, Kettle C, Thakar R. Methods of repair for obstetric anal sphincter injury. Cochrane Database Syst Rev. 2013;12: CD002866.

13. Buppasiri $P$, Lumbiganon $P$, Thinkhamrop J, Thinkhamrop B. Antibiotic prophylaxis for third- and fourth-degree perineal tear during vaginal birth. Cochrane Database Syst Rev. 2010;11:CD005125.

14. Duggal N, Mercado C, Daniels K, et al. Antibiotic prophylaxis for prevention of postpartum perineal wound complications: a randomized controlled trial. Obstet Gynecol. 2008;111(6):1268-1273.

15. Sultan AH, Thakar R, Fenner DE, editors. Perineal and Anal Sphincter Trauma. London: Springer; 2009.
International Journal of Women's Health

\section{Publish your work in this journal}

The International Journal of Women's Health is an international, peerreviewed open-access journal publishing original research, reports, editorials, reviews and commentaries on all aspects of women's healthcare including gynecology, obstetrics, and breast cancer. The manuscript management system is completely online and includes

\section{Dovepress}

a very quick and fair peer-review system, which is all easy to use. Visit http://www.dovepress.com/testimonials.php to read real quotes from published authors. 